\title{
Coordination of Nanoconjugation with an Antigen/Antibody for Efficient Detection of Gynecological Tumors
}

\author{
Xinmei Liu, ${ }^{1}$ Xinyuan Yang, ${ }^{2}$ Juan Shao, ${ }^{1}$ Yufeng Hong, ${ }^{3}$ Subash C. B. Gopinath $\mathbb{D D}^{4,5}$ \\ Yeng Chen, ${ }^{6}$ Mang Chek Wey, ${ }^{7}$ and Yaru Wang $\mathbb{D}^{8}$ \\ ${ }^{1}$ Department of Gynecology, The Fifth Hospital of Xi'an, Xi'an, Shaanxi 710082, China \\ ${ }^{2}$ Department of Gynecology, The First Affiliated Hospital of Xi'an Jiaotong University, Xi'an, Shaanxi 710061, China \\ ${ }^{3}$ Department of Gynecology, The Second Affiliated Hospital of Shaanxi University of Traditional Chinese Medicine, Xianyang, \\ Shaanxi 712000, China \\ ${ }^{4}$ School of Bioprocess Engineering, Universiti Malaysia Perlis, Arau 02600, Perlis, Malaysia \\ ${ }^{5}$ Institute of Nano Electronic Engineering, Universiti Malaysia Perlis, Kangar 01000, Perlis, Malaysia \\ ${ }^{6}$ Department of Oral \& Craniofacial Sciences, Faculty of Dentistry, University of Malaya, Kuala Lumpur 50603, Malaysia \\ ${ }^{7}$ Clinical Craniofacial Dental Research Group, Faculty of Dentistry, University of Malaya, Kuala Lumpur 50603, Malaysia \\ ${ }^{8}$ Department of Gynecology, Xi'an Maternal and Child Health Hospital, Xi'an, Shaanxi 710002, China
}

Correspondence should be addressed to Yaru Wang; wangyaru1966@sina.com

Received 7 September 2019; Revised 19 January 2020; Accepted 7 March 2020; Published 30 March 2020

Academic Editor: Bernd Hitzmann

Copyright $\odot 2020$ Xinmei Liu et al. This is an open access article distributed under the Creative Commons Attribution License, which permits unrestricted use, distribution, and reproduction in any medium, provided the original work is properly cited.

\begin{abstract}
Cervical, ovarian, and endometrial cancers are common in the female reproductive system. Cervical cancer starts from the cervix, while ovarian cancer develops when abnormal cells grow in the ovary. Endometrial or uterine cancer starts from the lining of the womb in the endometrium. Approximately 12,000 women are affected every year by cervical cancer in the United States. Squamous cell carcinoma antigen (SCC-Ag) is a well-established biomarker in serum for diagnosing gynecological cancers, and its levels were observed to be elevated in cervical, ovarian, and endometrial cancer patients. Moreover, SCC-Ag was used to identify the tumor size and progression stages. Various biosensing systems have been proposed to identify SCC-Ag; herein, enhanced interdigitated electrode sensing is presented with the use of gold nanoparticles (GNPs) to conjugate an antigen/antibody. It was proved that the limit of detection is $62.5 \mathrm{fM}$ in the case of antibody-GNP, which is 2-fold higher than that by SCC-Ag-GNP. Furthermore, the antibody-GNP-modified surface displays greater current increases with concomitant dose-dependent SCC-Ag levels. High analytical performance was shown by the discrimination against $\alpha$-fetoprotein and CYFRA 21-1 at 1 pM. An enhanced sensing system is established for gynecological tumors, representing an advance from the earlier detection methods.
\end{abstract}

\section{Introduction}

Gynecological tumors start from the cervix, endometrium, and ovaries of the female reproductive system, and the incidence has recently been increasing among the world population [1-3]. Accurate identification of gynecological tumors is the primary necessity to provide successful treatment for affected patients. Various sensing systems with different techniques have been used to identify the condition and progress of tumors [4-6]. Imaging techniques also support the identification of the advanced stages of gynecological tumors [7]. In addition, serum-based biomarker evaluation is a common strategy to confirm the presence of tumors and their stages [8-10]. Recently, cancer biomarkers have received increasing attention to indicate the level of tumors and the associated issues, even helping follow-up treatment responses [11-13]. It is critical to identify gynecological cancers such as ovarian, cervical, and endometrial cancers at earlier stages. Serum-based biomarkers help to identify the condition of diseases. Serumbased biomarkers originate from the tumor, can appear in the neighboring tissue, and are ultimately excreted into the 
blood. Moreover, tumor markers have been found to be secreted/released/leaked into the fluids in the interstitium, passing to the lymph and then the bloodstream $[11,13]$.

Squamous cell carcinoma antigen (SCC-Ag) is a glycoprotein that has been found to be a tumor antigen, showing elevated levels in gynecological tumors. Pretreatment of SCC was found to be related to the disease stage, tumor size, lymph node metastasis, and depth of stromal invasion. Additionally, increased levels of SCC-Ag were shown to have predictive value for tumor diagnosis $[6,7]$. The present work detected and quantified the level of SCC-Ag by its antibody for diagnosing gynecological cancer on an interdigitated electrode (IDE) sensing surface assisted by gold nanoparticle conjugation.

The application of nanomaterials in the field of biosensors has improved the detection of biomolecules and reduced the detection limit $[14,15]$. Different nanoparticles such as gold, silver, graphene, silica, and copper nanoparticles have been synthesized and used for various kinds of medical applications [16-18]. Among these materials, gold has unique optical properties and is well suited for the biosensor field to improve detection [19]. In general, gold has been used for surface modification/functionalization to immobilize or interact with probes or analyte molecules on sensing surfaces. In some cases, gold is conjugated with a detection probe or analyte molecule to enhance the sensitivity [20-22]. In this research, the conjugation of GNP with SCC-Ag (SCC-Ag-GNP) or antiSCC-Ag-antibody (SCC-Ag-antibody-GNP) on IDE sensing surfaces was compared.

\section{Materials and Methods}

2.1. Reagents and Biomolecules. SCC-Ag was obtained from RANDAX Life Sciences (Malaysia), anti-SCC-Ag antibody was purchased from Next Gene (Malaysia), ethanolamine, (3aminopropyl)triethoxysilane (APTES), ethanol, PBS (phosphate buffered saline), 16-mercaptoundecanoic acid, and human serum were obtained from Sigma-Aldrich (USA), and N-ethyl-N'-(3-dimethylaminopropyl)carbodiimide hydrochloride (EDC) and N-hydroxysuccinimide (NHS) were procured from GE Healthcare (USA). Gold nanoparticles with a size of $30 \mathrm{~nm}$ were purchased from Sigma-Aldrich (USA). $\alpha$-Fetoprotein and CYFRA 21-1 were from MyBioSource (USA). All other reagents used were of analytical grade.

2.2. IDE Sensing Surface Preparation. The fabrication of the IDE sensing surface was followed as stated in previous methods using different parameters with chemical and physical surface modifications [23]. Initially, the silicon wafers were oxidized at high temperature, and then, an etching process was carried out with aluminum. The followup processes were carried out as described earlier. The surface top layer was coated with zinc oxide. Before starting the surface chemical functionalization, the surface was washed with $1 \mathrm{M} \mathrm{KOH}$ (potassium hydroxide at $\mathrm{pH} 9.0$ ).

\subsection{Conjugation of Antibody/Antigen on the GNP Surface.} To immobilize the SCC-Ag-antibody on the GNP surface, the as-obtained GNPs were linked with $5 \mathrm{mM}$ 16-mercaptoundecanoic acid (16-MDA; contains both -SH and $-\mathrm{COOH}$ ends). Then, the samples were centrifuged at high speed to remove the excess 16-MDA. Next, the surface was activated and stabilized by NHS $(50 \mathrm{mM})$ and EDC (200 mM) at a ratio of $1: 1$. Afterwards, $200 \mathrm{nM}$ antibody was added to the activated surface of the GNPs and incubated for $1 \mathrm{~h}$ at RT. Then, the unbound antibodies were removed by centrifugation. The GNP-conjugated antibodies (SCC-Agantibody-GNP) were washed with $\mathrm{PBS}$ to completely remove unbound molecules and stored at $4^{\circ} \mathrm{C}$ for further use. Similar methods were used for the conjugation of SCC-Ag and GNPs (SCC-Ag-GNP). In this case, different concentrations of SCC-Ag were mixed with GNPs individually with the linker 16-MDA. The unbound SCC-Ag was removed by centrifugation, and SCC-Ag-GNP was used to detect its antibody.

2.4. Immobilization of SCC-Ag-Antibody-GNP on the IDE Sensing Surface. To detect SCC-Ag, we compared the antibody on the silane-modified surface of IDE with and without GNP conjugation. Initially, the IDE surface groups were converted to amine groups by dropping 3\% APTES diluted in $30 \%$ ethanol onto the surface and was kept at room temperature (RT) for $2 \mathrm{~h}$. Next, the amine-modified surface was washed thoroughly with $30 \%$ ethanol followed by water. Then, $200 \mathrm{nM}$ antibody with or without GNP was dropped on the amine-modified surface and kept for $30 \mathrm{~min}$ at RT to facilitate interactions between the amine surface and antibody/GNP. Finally, the remaining surface sites were blocked by dropping $1 \mathrm{M}$ ethanolamine to avoid any biofouling effects.

2.5. Diagnosis of SCC-Ag on SCC-Ag-Antibody Modified Surfaces. SCC-Ag was detected on the SCC-Ag-antibody immobilized surfaces by two methods. Method 1: (i) the surface groups were converted into amine groups by APTES; (ii) $200 \mathrm{nM}$ SCC-Ag-antibody was added; (iii) $1 \mathrm{M}$ ethanolamine was added; and (iv) SCC-Ag-GNP was added.

Method 2: (i) the surface groups were converted into amine groups by APTES; (ii) $200 \mathrm{nM}$ SCC-Ag-antibodyGNP was added; (iii) $1 \mathrm{M}$ ethanolamine was added; and (iv) SCC-Ag was added.

To check the detection limit, lower femtomolar to the lowest picomolar (62.5 fM to $1 \mathrm{pM}$ ) levels of SCC-Ag were dropped on the SCC-Ag-antibody-GNP immobilized surfaces from method 1 . In the case of method 2, the same concentration of SCC-Ag-GNP was dropped on the SCCAg-antibody immobilized surface for comparison. For the specificity analysis, $\alpha$-fetoprotein and CYFRA 21-1 were compared.

2.6. Spiking of SCC-Ag into Human Serum and Detection on an Antibody-GNP-Modified Surface. To determine the ability of detecting SCC-Ag in a real biological sample and perform competition experiments, SCC-Ag was spiked into human serum and detected by antibody-GNP conjugates. For that purpose, SCC-Ag with concentrations from 30 to $240 \mathrm{fM}$ 


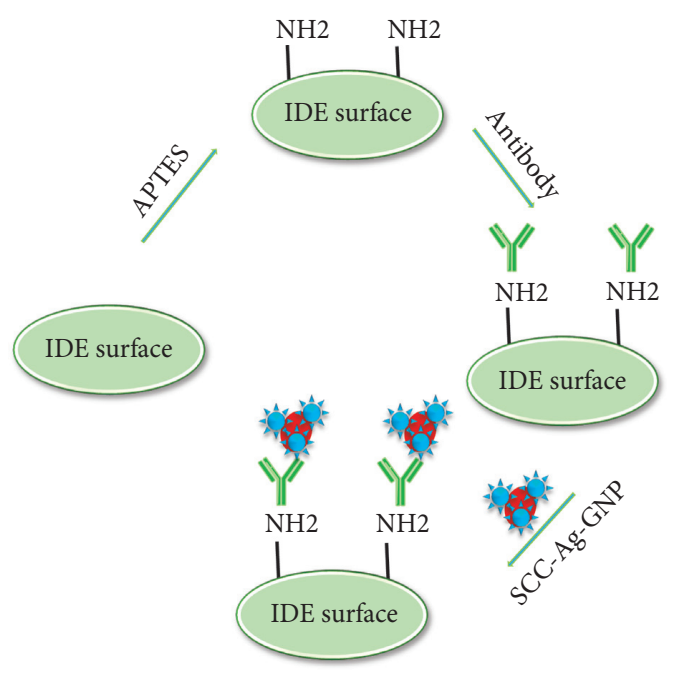

(a)

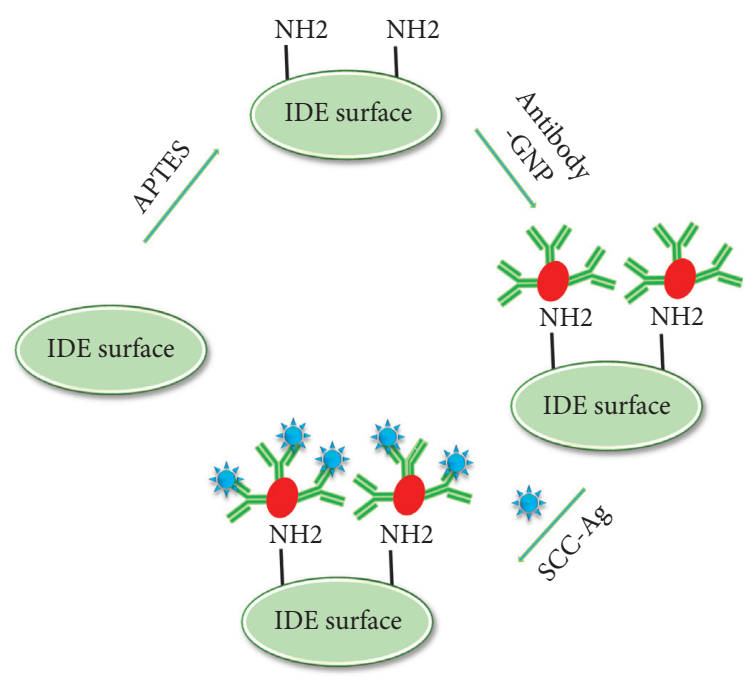

(b)

FIGURE 1: Schematic representation of the detection of SCC-Ag on the amine-modified IDE surface. (a) SCC-Ag-GNP detection on the SCC-Ag antibody-modified surface. The antibody was immobilized on the APTES-modified surface, and then, SCC-Ag-GNP interacted with the antibody. (b) SCC-Ag-antibody-GNP was immobilized on the APTES-modified surface, and then, SCC-Ag interacted on the surface, enabling its detection.

was spiked and dropped on an antibody-GNP-conjugated IDE sensing surface. The changes in the current were noted to detect SCC-Ag.

\section{Results and Discussion}

Identifying gynecological tumors, such as ovarian, cervical, and endometrial cancers, is mandatory to treat these diseases and avoid spreading to other organs of the body. Serumbased biomarkers help to identify tumors effectively and are considered to have diagnostic potential. SCC-Ag is one of the proven biomarkers in serum for tumors, and its concentration is elevated in most gynecological tumors [24]. In this work, SCC-Ag was detected by assistance with its antibody on an amine-modified IDE sensing surface. IDE sensors have been shown to efficiently detect various diseases with highly specific interactions of biomolecules [25, 26]. To improve the detection limit, a gold nanoparticle- (GNP-) conjugated antibody or GNP-conjugated SCC-Ag was used, and these two methods were compared on the IDE sensing surfaces. GNPs have been used in different ways to improve diagnostic systems, mainly by surface modification and conjugation with analytes or target molecules. Surface modification helps to evenly arrange molecules on the sensing surface and helps increase the number of biomolecules immobilized on the surface of the sensor. Since it has been proven that the proper arrangement of biomolecules on the surface of the sensor improves the detection system [27, 28], we also expected the proper arrangement of biomolecules with the help of GNPs to improve the detection of SCC-Ag. In the other case, when the detection molecule is conjugated with GNPs, a higher number of biomolecules bind to the GNP surfaces, so in this work, the conjugation of SCC-Ag to GNPs improves the binding of its antibody on the IDE surface. The above two methods were compared with similar concentrations of SCC-Ag. Figure 1 shows a schematic representation of the detection strategy for SCC-Ag on amine-modified surfaces. In the case of method 1, SCC-Ag-antibody was immobilized on the amine-IDE surface, and then, SCC-Ag-GNP was used to detect the level of SCC-Ag (Figure 1(a)). Figure 1(b) explains method 2 for the detection of SCC-Ag on the SCCAg-antibody-GNP-immobilized surface. The SCC-Ag-antibody-GNP was bound on the sensing surface by amine interactions, and then, SCC-Ag could interact; this happens because the GNPs, which have a negative surface charge, bind on the positively charged amine-modified surfaces by electrostatic interactions [29].

3.1. Comparison of Antibody Immobilization on the IDE Surface with and without GNPs. As explained above, two different kinds of probe modifications were prepared on the IDE surface by SCC-Ag-antibody: with and without GNPs. As shown in Figure 2(a), with the bare surface, the maximum current level was $2.66 E^{-06}$; when APTES was added on the surface, the current was increased to $4.08 E^{-06}$. This result confirms the amine modification on the IDE surface. Next, when $200 \mathrm{nM}$ antibody was dropped on the surface, the current was increased to $5.86 E^{-06}$ and gave a difference of $1.78 E^{-06}$, and then, $1 \mathrm{M}$ ethanolamine, as a blocking agent, caused the current to increase to $6.52 E^{-06}$. These results clearly show the proper binding of the probe SCC-Ag-antibody on the IDE surface. In the case of method 2, after APTES treatment, $200 \mathrm{nM}$ antibody-GNP was added, and the current increased from $3.18 E^{-06}$ to $5.05 E^{-06}$ (Figure 2(b)). Thus, the current difference was $1.87 E^{-06}$, which is an almost 1.6-fold increase compared with the current change when conjugating the antibody with GNPs. This increase is due to the larger number of antibodies 


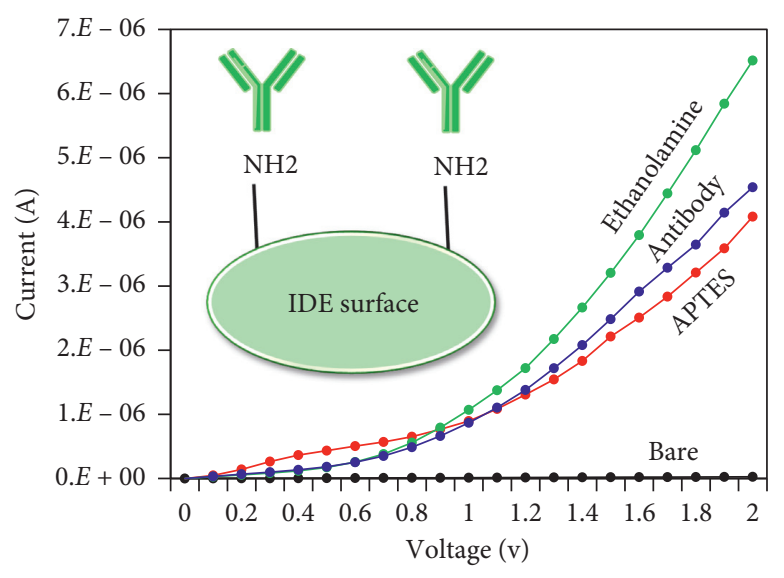

(a)

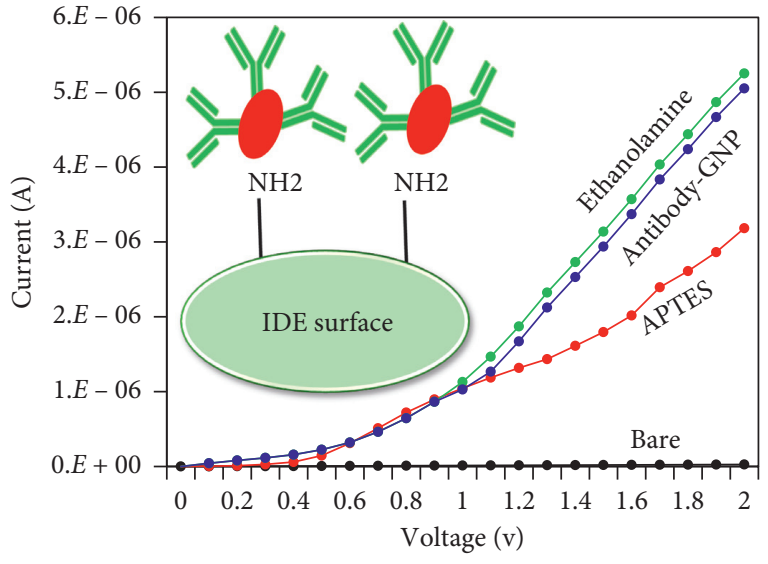

(b)

FIGURE 2: Comparison of the antibody immobilization process on APTES-modified IDE sensing surfaces: (a) without GNP; (b) with GNP. Antibody-GNP-modified surfaces show greater changes in current increases than those without GNPs.

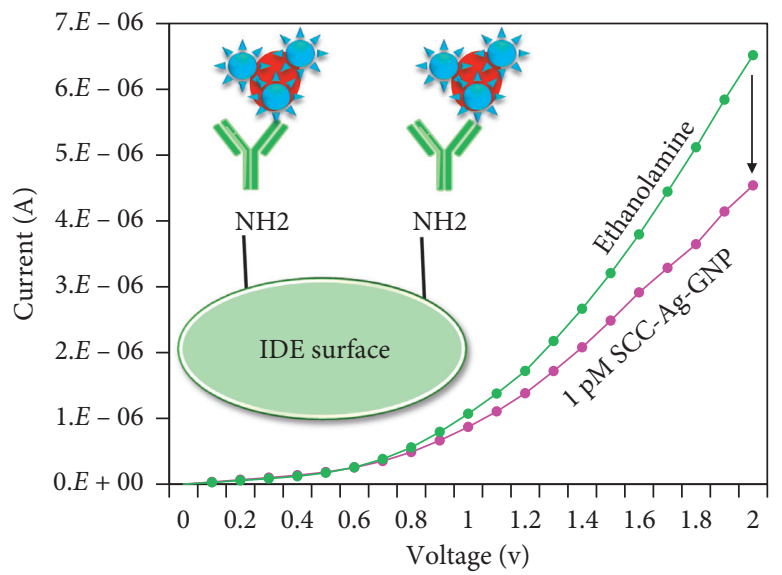

(a)

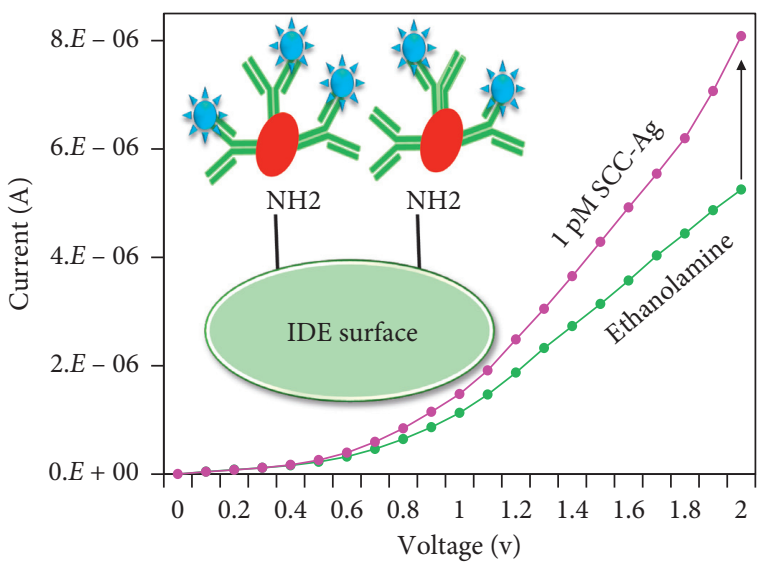

(b)

FIgUre 3: Detection of 1 pM SCC-Ag by the methods: (a) SCC-Ag-GNP with the antibody; (b) SCC-Ag on the SCC-Ag-antibody-GNP surface.

immobilized on the surface of GNPs, which leads to more immobilized antibodies on the IDE surface for the sensing interactions.

\subsection{Detection by Conjugation of SCC-Ag-Antibody-GNP/} SCC-Ag-GNP: Comparison. It was proved that SCC-Agantibody-GNP shows higher current changes than SCCAg-antibody does. Then, the detection of SCC-Ag on SCC-Ag-antibody-modified IDE sensing surfaces (method 1) and SCC-Ag-antibody-GNP-modified IDE sensing surfaces (method 2) was performed. Figure 3(a) shows $1 \mathrm{pM}$ SCC-Ag-GNP detection on the antibody-immobilized surface, clearly showing a current change from $6.52 E-06$ to $4.54 E-06$ with a difference of $1.98 E^{-06}$. In the case of method 2 , the similar concentration of 1 pM SCC-Ag on the SCCAg-antibody-GNP-immobilized surface shows a current increase from $5.25 E-06$ to $8.08 E-06$, and the difference was found to be $2.83 E-06$ (Figure $3(\mathrm{~b})$ ). From this result, it was concluded that method 2 (SCC-Ag on SCC-Ag-antibodyGNP) shows greater changes than method 1 (SCC-Ag-GNP on SCC-Ag-antibody). This result might be due to the larger number of probes available with SCC-Ag-antibody-GNP bound on the APTES-modified IDE surface, leading to a larger amount of SCC-Ag binding.

3.3. Limit of SCC-Ag Detection. The limit of SCC-Ag detection was also determined with both methods 1 and 2 . For this assessment, SCC-Ag was titrated from $62.5 \mathrm{fM}$ to $1 \mathrm{pM}$ and detected by both methods 1 and 2. In the case of method 1 , with a $62.5 \mathrm{fM}$ concentration, the current showed a change from $6.52 E-06$ to $6.33 E-06$. With increasing concentrations of SCC-Ag, the concomitant current levels gradually decreased. At $125 \mathrm{fM}$ SCC-Ag, the current was 6.06E-06; at $250 \mathrm{fM}$, it was $5.38 E-06$; at $500 \mathrm{fM}$, it was $4.92 E-06$; and at $1 \mathrm{pM}$, it was $4.54 E-06$. (Figure 4(a)). In the case of method 2, at $62.5 \mathrm{fM}$ SCC-Ag, the current increased from $5.25 E-06$ to 


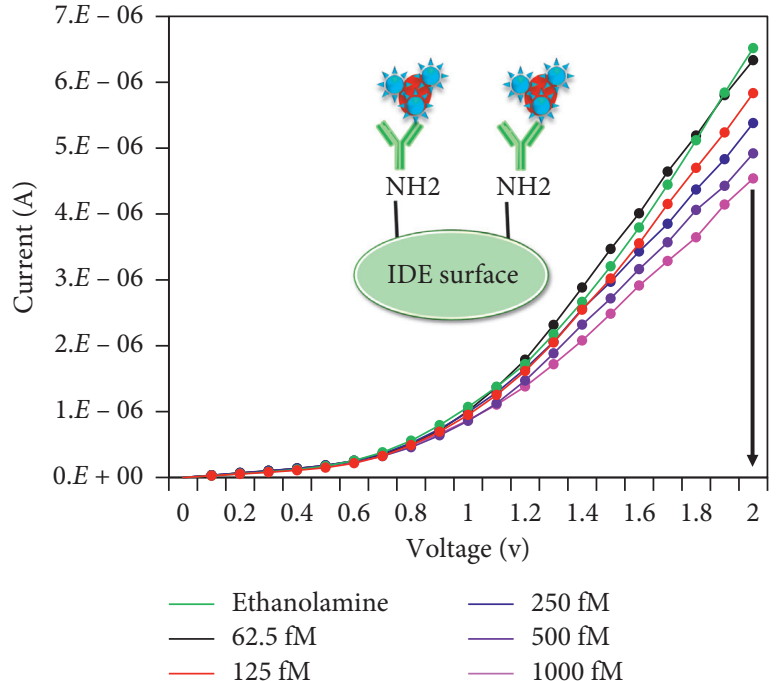

(a)

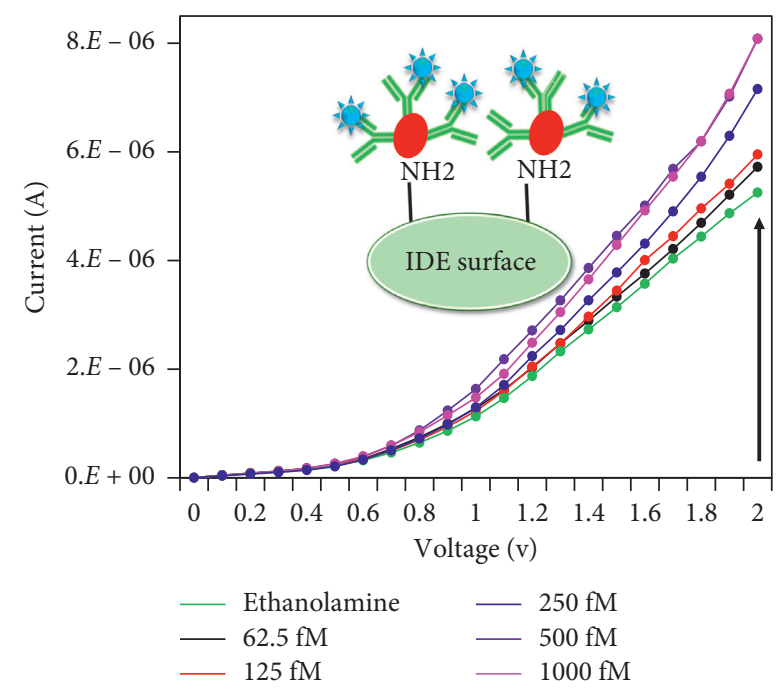

(b)

FIGURE 4: Limit of SCC-Ag detection. (a) SCC-Ag-GNP at concentrations from $62.5 \mathrm{fM}$ to $1 \mathrm{pM}$ was detected with the antibody. (b) SCC-Ag at concentrations from $62.5 \mathrm{fM}$ to $1 \mathrm{pM}$ was detected on antibody-GNP surfaces. SCC-Ag-antibody-GNP-modified surfaces show a greater response of current at all concentrations of SCC-Ag tested.

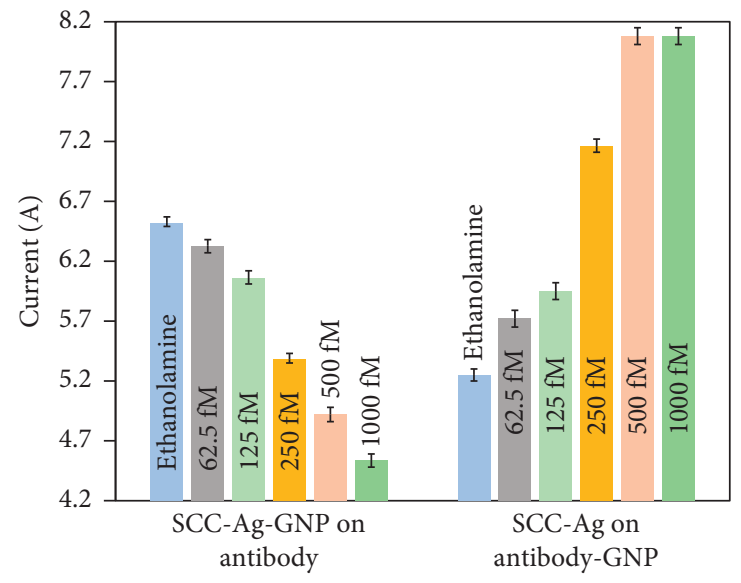

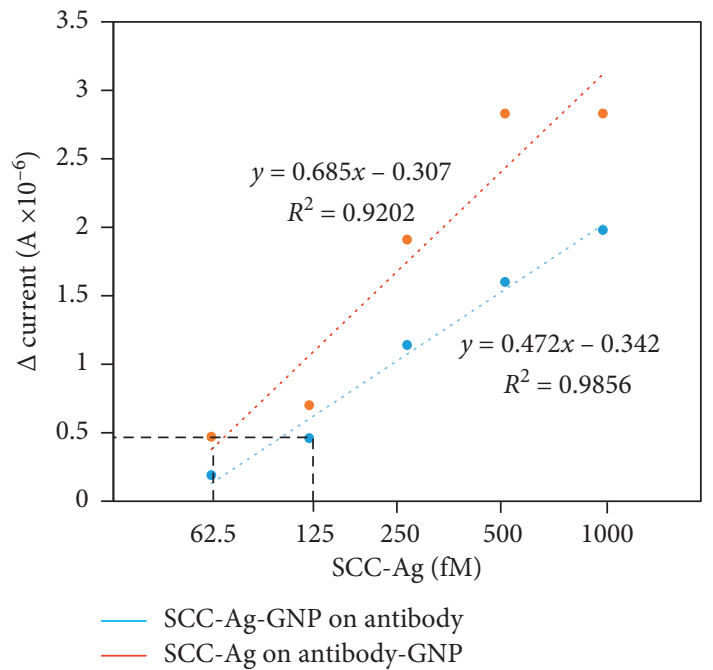

(b)

FIgURE 5: (a) Comparison of the difference in current changes with various concentrations of SCC-Ag. Methods of SCC-Ag-GNP on the antibody and SCC-Ag on SCC-Ag-antibody-GNP surfaces were considered. Both methods show significant changes in the current from $62.5 \mathrm{fM}$ and saturated at $1 \mathrm{pM}$ SCC-Ag. (b) Linear regression analysis for the interaction of different concentrations of SCC-Ag-GNP with ASC-Ag-antibody and SCC-Ag with SCC-Ag-antibody-GNP. The sensitivity was calculated based on $3 \sigma$ as indicated. The calculated sensitivities are 125 and $62.5 \mathrm{fM}$ for methods 1 and 2 , respectively.

$5.72 E-06$; at $125 \mathrm{fM}$, it was $5.95 E-06$; at $250 \mathrm{fM}$, it was $7.16 E-06$; and at $500 \mathrm{fM}$ and $1 \mathrm{pM}$, a saturation current of $8.08 E-06$ was observed (Figure 4(b)). Comparing methods 1 and 2, method 2 shows greater changes in the current increase at all the concentrations of SCC-Ag tested.

Figure 5(a) shows the comparison of the difference in current changes with all the concentrations of SCC-Ag by methods 1 and 2 . It was noticed that compared with method 1 , method 2 showed gradual increases in the current changes at all the tested concentrations of SCC-Ag. This is in another result from method 1 , which might be due to the proper arrangement of SCC-Ag-antibody-GNP on the IDE sensing surface. In method 2, the apparent current change was noticed from $60 \mathrm{fM}$, while in method 1, it was noticed from $120 \mathrm{fM}$. Figure 5(b) presents a linear regression analysis of the interaction of different concentrations of SCC-Ag with its antibody; the sensitivity was calculated based on $3 \sigma$. It was clear by these analyses that the sensitivity attained by 


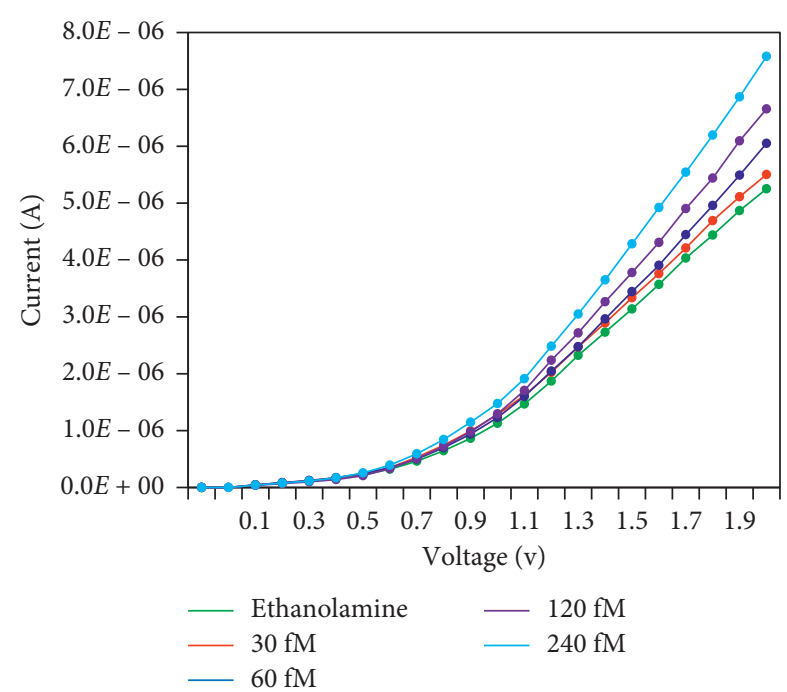

FIgURE 6: Spiking of SCC-Ag into human serum. SCC-Ag concentrations from 30 to $250 \mathrm{fM}$ were spiked in human serum and detected by SCC-Ag-GNP. Apparent changes were noted in comparison with the condition of spiking into PBS.

Table 1: Comparison of methods for the detection of SCC-Ag.

\begin{tabular}{lcc}
\hline Detection method & $\begin{array}{c}\text { Limit of } \\
\text { detection }\end{array}$ & Reference \\
\hline Electrochemical sensor & $10 \mathrm{pM}$ & {$[24]$} \\
Electrochemiluminescent sensor & $0.4 \mathrm{pg} / \mathrm{mL}$ & {$[30]$} \\
Surface plasmon resonance & $0.1 \mathrm{pM}$ & {$[31]$} \\
Surface-enhanced Raman & $7.16 \mathrm{pg} / \mathrm{mL}$ & {$[32]$} \\
scattering & $80 \mathrm{pM}$ & {$[33]$} \\
Electrochemical sensor & $10 \mathrm{fg} / \mathrm{mL}$ & {$[34]$} \\
Field effect transistor & $10 \mathrm{fM}$ & Current \\
Interdigitated electrode sensor & & study \\
\hline
\end{tabular}

methods 1 and 2 was 120 and $62.5 \mathrm{fM}$, respectively. These ranges are comparable, showing better performance than that of the currently available sensors (Table 1).

3.4. Detection of SCC-Ag-Spiked Human Serum on the Antibody-GNP-Modified IDE Sensing Surface. After confirming the SCC-Ag detection limit, to evaluate the ability of SCC-Ag detection in the biological sample, different concentrations of SCC-Ag were spiked into human serum and detected by antibody-GNP conjugates. As shown in Figure 6, when $30 \mathrm{fM}$ SCC-Ag was spiked in serum, the current did not significantly change, but the change was better than that of the SCC-Ag-spiked PBS sample. When the concentration was increased to $60 \mathrm{fM}$, the current clearly increased. Furthermore, with increasing concentrations of SCC-Ag, the current levels also gradually increased. As a well-known fact, serum has large quantities of proteins and biomarkers. Albumin and globulin are the predominant proteins in the serum, at $45 \mathrm{mg} \cdot \mathrm{mL}^{-1}$ and
20-35 mg.mL $\mathrm{m}^{-1}$, respectively. In addition, the commonly recognized IgM level is $0.75-3.0 \mathrm{mg} \cdot \mathrm{mL}^{-1}$, and the $\operatorname{IgG}$ level is $6.5-18.50 \mathrm{mg} \cdot \mathrm{mL}^{-1}$. Considering these higher levels of interferents/competitors, the above assay is competition-based. It has been reported that an SCC-Ag level of $2 \mathrm{ng} / \mathrm{mL}$ is the upper limit of normal individuals, and the current method offering lower to higher levels of detection of SCC-Ag helps to distinguish between normal and cancer patients.

\section{Conclusion}

Gynecological tumors in the female reproductive system mainly occur in the form of cervical, ovarian, and endometrial cancers. They cause various health issues, and the later stage of these tumors spread to other parts of the body, making it mandatory to identify the tumor at earlier stages. Early diagnosis will help improve treatment and avoid metastasis. Squamous cell carcinoma antigen (SCC- $\mathrm{Ag}$ ) is a serum-based biomarker that has been found at elevated levels in gynecological tumors. In this work, SCC-Ag was detected on an amine-modified interdigitated electrode sensor assisted by the antibody. Gold nanoparticle-conjugated biomolecules were used to improve the detection. Two methods, namely, SCC-Ag-GNP on SCC-Ag-antibody (method 1) and SCC-Ag on SCC-Ag-antibody-GNP (method 2), were compared for detection. It was found that method 2 shows better sensitivity with a higher increase in current changes at all concentrations of SCC-Ag tested and worked well in the SCC-Ag-spiked serum samples. Such methods with gold-conjugated probes/targets will help to identify and quantify the severity level of gynecological tumors.

\section{Data Availability}

All the data and materials are available without restriction.

\section{Conflicts of Interest}

The authors declare that they have no conflicts of interest.

\section{Authors' Contributions}

Xinmei Liu contributed to conceptualization, methodology, data analysis, writing and preparing the original draft, and investigation. Xinyuan Yang contributed to conceptualization, investigation, validation, visualization, reviewing, and editing. Juan Shao contributed to investigation, validation, visualization, reviewing, and editing. Yufeng Hong was responsible for data analysis, reviewing, and editing. Subash C. B. Gopinath contributed to validation, reviewing, and editing. Yeng Chen expanded the methodology and contributed to reviewing and editing. Wey Mang Chek expanded the methodology and contributed to reviewing and editing. Yaru Wang was responsible for conceptualization, methodology, data analysis, supervision, writing and preparing the original draft, and investigation. 


\section{References}

[1] Y. L. Woo, M. Kyrgiou, A. Bryant, T. Everett, and H. O. Dickinson, "Centralisation of services for gynaecological cancer," Cochrane Database of Systematic Reviews, vol. 3, Article ID CD007945, 2012.

[2] K. Elmasry and S. A. Gayther, "Genetic mutations in gynaecological cancers," Reviews in Gynaecological and Perinatal Practice, vol. 6, no. 3-4, pp. 115-125, 2006.

[3] G. S. Kienle, A. Glockmann, M. Schink, and H. Kiene, "Viscum album L. extracts in breast and gynaecological cancers: a systematic review of clinical and preclinical research," Journal of Exerimental and. Clinical Cancer Research, vol. 28, no. 1, p. 79, 2009.

[4] S. A. O'Toole, B. L. Sheppard, E. P. J. McGuinness, N. C. Gleeson, M. Yoneda, and J. Bonnar, "The MTS assay as an indicator of chemosensitivity/resistance in malignant gynaecological tumours," Cancer Detection and Prevention, vol. 21, no. 1, pp. 47-54, 2003.

[5] C. Pyragius, M. Fuller, C. Ricciardelli, and M. Oehler, "Aberrant lipid metabolism: an emerging diagnostic and therapeutic target in ovarian cancer," International Journal of Molecular Sciences, vol. 14, no. 4, pp. 7742-7756, 2013.

[6] M. Takakura, S. Kyo, M. Nakamura et al., "Circulating tumour cells detected by a novel adenovirus-mediated system may be a potent therapeutic marker in gynaecological cancers," British Journal of Cancer, vol. 107, no. 3, pp. 448-454, 2012.

[7] M. A. Queiroz, R. A. Kubik-Huch, N. Hauser et al., "PET/MRI and PET/CT in advanced gynaecological tumours: initial experience and comparison," European Radiology, vol. 25, no. 8, pp. 2222-2230, 2015.

[8] J. Ferlay, I. Soerjomataram, R. Dikshit et al., "Cancer incidence and mortality worldwide: sources, methods and major patterns in GLOBOCAN 2012," International Journal of Cancer, vol. 136, no. 5, pp. 359-386, 2015.

[9] R. E. Brand, B. M. Nolen, H. J. Zeh et al., "Serum biomarker panels for the detection of pancreatic cancer," Clinical Cancer Research, vol. 17, no. 4, pp. 805-816, 2011.

[10] P. R. Srinivas, B. S. Kramer, and S. Srivastava, "Trends in biomarker research for cancer detection," The Lancet Oncology, vol. 2, no. 11, pp. 698-704, 2001.

[11] E. Anastasi, S. Gigli, L. Ballesio, A. Angeloni, and L. Manganaro, "The complementary role of imaging and tumor biomarkers in gynecological cancers: an update of the literature," Asian Pacific Journal of Cancer Prevention, vol. 19, no. 2, pp. 309-317, 2018.

[12] Y. Ueda, T. Enomoto, T. Kimura et al., "Serum biomarkers for early detection of gynecologic cancers," Cancers, vol. 2, no. 2, pp. 1312-1327, 2010.

[13] R. Molina, J. M. Escudero, J. M. Augé et al., "HE4 a novel tumour marker for ovarian cancer: comparison with CA 125 and ROMA algorithm in patients with gynaecological diseases," Tumor Biology, vol. 32, no. 6, pp. 1087-1095, 2011.

[14] I. Letchumanan, M. K. Md Arshad, S. R. Balakrishnan, and S. C. B. Gopinath, "Gold-nanorod enhances dielectric voltammetry detection of c-reactive protein: a predictive strategy for cardiac failure," Biosensors and Bioelectronoics, vol. 130, pp. 40-47, 2019.

[15] T. Lakshmipriya, M. Fujimaki, S. C. B. Gopinath, K. Awazu, Y. Horiguchi, and Y. Nagasaki, "A high-performance waveguide-mode biosensor for detection of factor IX using PEGbased blocking agents to suppress non-specific binding and improve sensitivity," The Analyst, vol. 138, no. 10, p. 2863, 2013.
[16] M. E. Foo and S. C. B. Gopinath, "Feasibility of graphene in biomedical applications," Biomedecine and Pharmacotherapy, vol. 94, pp. 354-361, 2017.

[17] J. N. Tiwari, V. Vij, K. C. Kemp, and K. S. Kim, "Engineered carbon-nanomaterial-based electrochemical sensors for biomolecules," ACS Nano, vol. 10, no. 1, pp. 46-80, 2016.

[18] F. Wang, T. Lakshmipriya, and S. C. B. Gopinath, "Red spectral shift in sensitive colorimetric detection of tuberculosis by ESAT-6 antigen-antibody Complex : a new strategy with gold nanoparticle," Nanoscale Research Letter, vol. 13, no. 1, p. 331, 2018.

[19] J. Nagel, P. Chunsod, C. Zimmerer, F. Simon, A. Janke, and G. Heinrich, "Immobilization of gold nanoparticles on a polycarbonate surface layer during molding," Materials Chemistry and Physics, vol. 129, no. 1-2, pp. 599-604, 2011.

[20] T. Lakshmipriya, S. C. B. Gopinath, and T.-H. Tang, "BiotinStreptavidin competition mediates sensitive detection of biomolecules in enzyme linked immunosorbent assay," PLoS One, vol. 11, no. 3, Article ID e0151153, 2016.

[21] S. C. B. Gopinath, T. Lakshmipriya, and K. Awazu, "Colorimetric detection of controlled assembly and disassembly of aptamers on unmodified gold nanoparticles," Biosensors and Bioelectronics, vol. 51, pp. 115-123, 2014.

[22] T. Lakshmipriya, S. C. B. Gopinath, M. Citartan, U. Hashim, and T.-H. Tang, "Gold nanoparticle-mediated high-performance enzyme-linked immunosorbent assay for detection of tuberculosis ESAT-6 protein," Micro and Nanosystems, vol. 8, no. 2, pp. 92-98, 2017.

[23] I. Letchumanan, S. C. B. Gopinath, M. K. Md Arshad, P. Anbu, and T. Lakshmipriya, "Gold nano-urchin integrated label-free amperometric aptasensing human blood clotting factor IX: a prognosticative approach for "Royal disease"” Biosensors and Bioelectronics, vol. 131, pp. 128-135, 2019.

[24] H. Wang, T. Lakshmipriya, Y. Chen, and S. C. B. Gopinath, "Squamous cell carcinoma biomarker sensing on a strontium oxide-modified interdigitated electrode surface for the diagnosis of cervical cancer," Biomedical Research International, vol. 2019, Article ID 2807123, 7 pages, 2019.

[25] Q. Lv, Y. Wang, C. Su et al., "Human papilloma virus DNAbiomarker analysis for cervical cancer: signal enhancement by gold nanoparticle-coupled tetravalent streptavidin-biotin strategy," International Journal of Biological Macromolecules, vol. 134, pp. 354-360, 2019.

[26] N. Z. Jin, S. Anniebell, S. C. B. Gopinath, and Y. Chen, "Variations in spontaneous assembly and disassembly of molecules on unmodified gold nanoparticles," Nanoscale Research Letters, vol. 11, no. 1, p. 399, 2016.

[27] Y. Nagasaki, "Construction of a densely poly(ethylene glycol)chain-tethered surface and its performance," Polymer Journal, vol. 43, no. 12, pp. 949-958, 2011.

[28] T. Lakshmipriya, Y. Horiguchi, and Y. Nagasaki, "Coimmobilized poly(ethylene glycol)-block-polyamines promote sensitivity and restrict biofouling on gold sensor surface for detecting factor IX in human plasma," The Analyst, vol. 139, no. 16, pp. 3977-3985, 2014.

[29] S. Williams, P. Davies, J. Bowen, and C. Allender, "Controlling the nanoscale patterning of AuNPs on silicon surfaces," Nanomaterials, vol. 3, no. 1, pp. 192-203, 2013.

[30] L. Wu, Y. Hu, Y. Sha et al., "An "in-electrode"-type immunosensing strategy for the detection of squamous cell carcinoma antigen based on electrochemiluminescent AuNPs/g-C 3 N 4 nanocomposites," Talanta, vol. 160, pp. 247-255, 2016.

[31] Q. Zhao, R. Duan, J. Yuan, Y. Quan, H. Yang, and M. Xi, “A reusable localized surface plasmon resonance biosensor for 
quantitative detection of serum squamous cell carcinoma antigen in cervical cancer patients based on silver nanoparticles array," International Journal of Nanomedicine, vol. 9, no. 1, pp. 1097-1104, 2014.

[32] D. Lu, J. Xia, Z. Deng, and X. Cao, "Detection of squamous cell carcinoma antigen in cervical cancer by surface-enhanced Raman scattering-based immunoassay," Analytical Methods, vol. 11, no. 21, pp. 2809-2818, 2019.

[33] T. Liang, Q. Qu, Y. Chang, S. C. B. Gopinath, and X. T. Liu, "Diagnosing ovarian cancer by identifying SCC-antigen on a multiwalled carbon nanotube-modified dielectrode sensor," Biotechnology and Applied Biochemistry, vol. 66, no. 6, pp. 839-944, 2019.

[34] Y. Zhang, R. Chen, L. Xu, Y. Ning, S. Xie, and G.-J. Zhang, "Silicon nanowire biosensor for highly sensitive and multiplexed detection of oral squamous cell carcinoma biomarkers in saliva," Analytical Sciences, vol. 31, no. 2, pp. 73-78, 2015. 